\title{
On Nonlinear Control Perspectives of a Challenging Benchmark
}

\author{
Guangyu Liu and Yanxin Zhang \\ The University of Auckland \\ New Zealand
}

\section{Introduction}

Dynamical systems are often nonlinear in nature. It motives people to explore various theoretical nonlinear analysis and control design tools, of which constructive nonlinear design methods are the most celebrated ones. However, applying a constructive tool faces up a big hurdle that the tool deals only with a certain dynamical structure, often not possessed by the natural dynamics. Nonlinear constructive control designs heavily relies on the identification of a particular structure via coordinate transformation and control transformation. To be realistic, these theoretical tools are not general to all of the nonlinear systems. Here, a challenging benchmark example-a four degrees of freedom inverted pendulum under the influence of a planar force-is considered that is nonlinear, multiple input and multiple output, underactuated and unstable. The benchmark is also of practical interests because it is an abstract of several applications. Three challenging control objectives are envisaged for the first time in the literature in order to how to apply various cuttingedge theoretical nonlinear control tools. In fact, the key step of all of the nonlinear designs is to identify spectral structures- certain "normal" forms. From this aspect, a sequence of preliminary designs will accompany the existing tools to construct nonlinear controllers, which is quite different from the linear control designs.

\section{The benchmark problem}

\subsection{Modeling}

The spherical inverted pendulum is subject to a holonomic constraint on the vertical direction and its self-spin about the principal axis along the pole is neglected from the context. As a result, the benchmark has only four degrees of freedom described by a set of generalized coordinates $q \in R^{4}$ that include two translational ones (also called external variables) and two angular ones (also called shape variables). The translational coordinates are unanimously denoted by two globally fixed Cartesian coordinates $(x, y)$ while the angular ones have several choices as is given later. $Q \in R^{4}$ denotes the generalized input for the system with

$$
Q=\left(F_{x}, F_{y}, 0,0\right)^{T}+v_{f},
$$

where $\left(F_{x}, F_{y}\right) \triangleq F$ is the actual planar force and $v_{f} \in R^{4}$ is a collection of exogenous disturbances and unmodelled dynamics. 


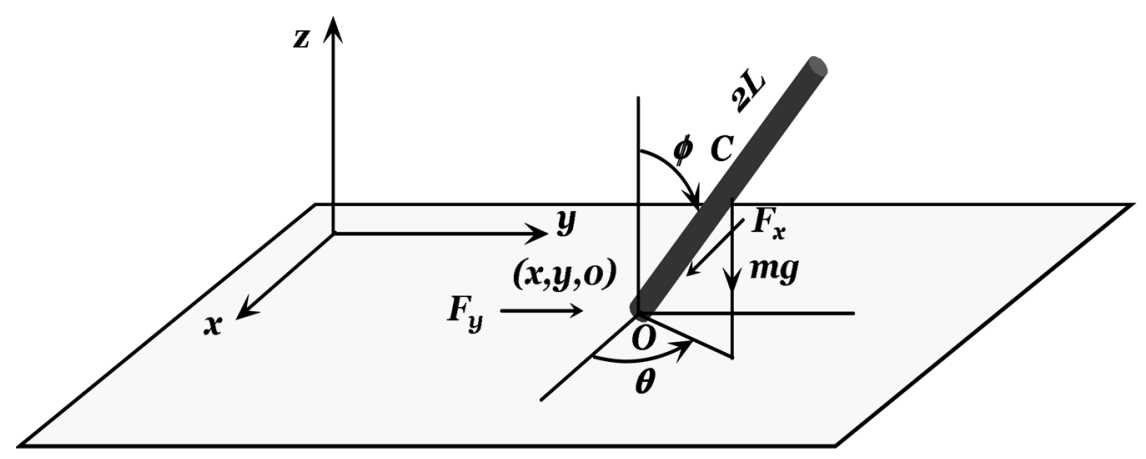

M.1

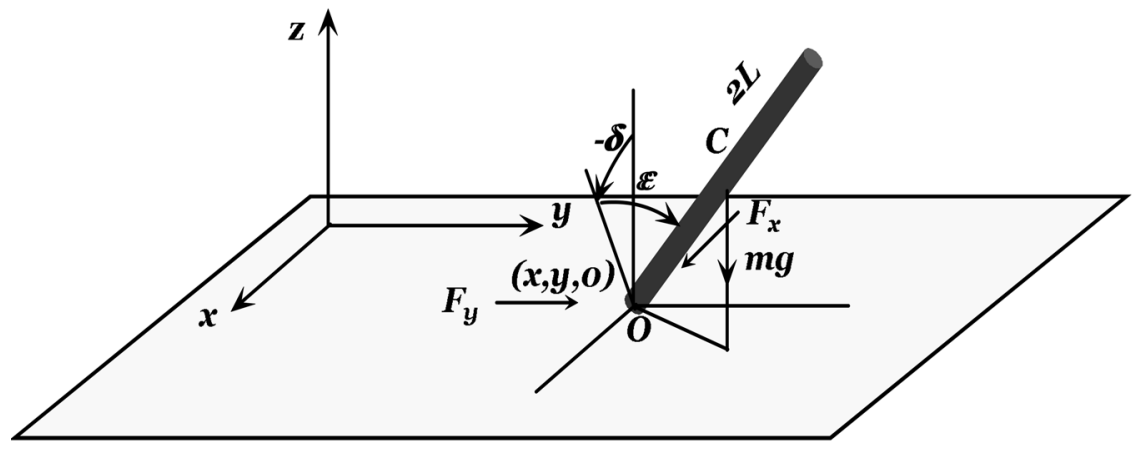

M.2

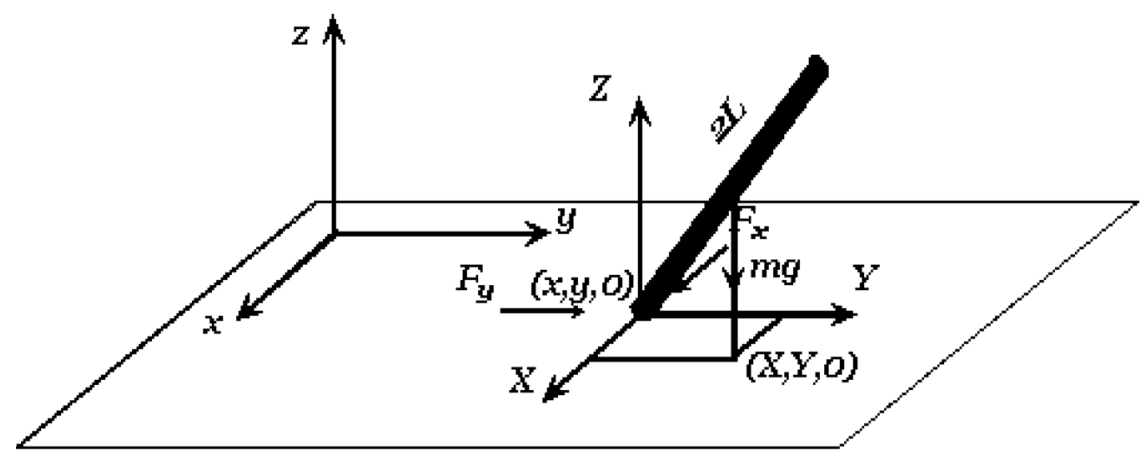

M.3

Fig. 1. The configurations of a spherical inverted pendulum 
Define a Lagrangian $\mathcal{L}=K-V$ where $K$ and $V$ are respectively the kinetic energy and the potential energy of the benchmark. Applying the Euler-Lagrangian equations

$$
\frac{d}{d t} \frac{d \mathcal{L}}{d \dot{q}}-\frac{d \mathcal{L}}{d q}=Q
$$

for the benchmark derives the dynamics

$$
\mathbf{D}(q) \cdot\{\ddot{q}\}+\mathbf{C}(q, \dot{q}) \cdot\left\{\dot{q}_{i}\right\}+\mathbf{G}(q)=Q,
$$

where $\mathbf{D}(q)$ is the matrix of inertia, $\mathbf{C}(q, \dot{q})$ is the centrifugal and Coriolis matrix and $\mathbf{G}(q)$ is the gravitational matrix. Equation 3 is taken as the mathematical model of the benchmark. Three models with respect to three sets of generalized coordinates are derived (see Fig. 1)

M.1 The model in $\mathrm{q}=(x, y, \theta, \phi)$ in (Liu, 2006) $-\theta$ and $\phi$ are the procession and nutation angles respectively; the model has singular points at $\phi=\ldots, 0, \pi, 2 \pi, \ldots$ but the model is ideal for the objective of swing-up (e.g., (Albouy \& Praly, 2000)); the upper space is defined by $U=\left\{(x, y, \theta, \phi, \dot{x}, \dot{y}, \dot{\theta}, \dot{\phi}) \in R^{8} \mid-\pi / 2<\phi<\pi / 2\right\} ;$

M.2 The model in $\mathrm{q}=(x, y, \delta, \epsilon)$ in (Liu et al., 2008a) $-\delta$ and $\varepsilon$ denote the heading and bank angles respectively; the model has singular points at $\delta=\pi / 2,3 \pi / 2, \ldots$ and/or $\epsilon=$ $\pi / 2,3 \pi / 2, \ldots$ that does not affect the control objectives here; special structures have been derived from this model (see S.1 and S.2 in the sequel); the upper space is defined by $U=\left\{(x, y, \delta, \epsilon, \dot{x}, \dot{y}, \dot{\delta}, \dot{\epsilon}) \in R^{8} \mid-\pi / 2<\delta<\pi / 2\right.$ and $\left.-\pi / 2<\epsilon<\pi / 2\right\} ;$

M.3 The model in $q=(x, y, X, Y)$ in (Liu et al., 2008b) $-X$ and $Y$ are the projection of the center of mass in the horizontal plane; the model can only represent the case that the pendulum is either above the horizontal plane or below the plane but it is sufficient to the control objectives in this paper; the description of the model is technically simpler than the above two but we cannot ensure that it also implies particular structures as those derived from M.2; the upper space is defined by $U=\{(x, y, X, Y, \dot{x}, \dot{y}, \dot{X}, \dot{Y}) \in$ $\left.R^{8} \mid \sqrt{X^{2}+Y^{2}}<L\right\}$ ( $L$ is the length of the center of mass to the pivot).

Generally, Equation 3 can be written in a state space form

$$
\dot{\eta}=f\left(\eta, F, v_{f}\right)
$$

where $\eta \triangleq(q, \dot{q}) \in U$ denotes the state vector and Equation 4 is called the nominal dynamics as $v_{f} \equiv 0$.

\subsection{Problem formulation}

In the literature, a local stabilizing controller is used to switch from a swing-up strategy (Albouy \& Praly, 2000) to achieve a large domain attraction. Here, three different control objectives are envisaged which are more challenging:

PF.1 The non-local stabilization - Find a planar force $F$ to drive the spherical inverted pendulum in such a way that for a non-trivial set $S \subset U$ and $S \ni 0$, where the trivial solution denotes the upright position of the pendulum and a given point on the horizontal plane in $(x, y)$ for the universal joint of the pendulum, $S$ is contained in a domain of attraction. If $S \subseteq U$ and $U \subseteq S$, the closed loop system is said to yield a "global" stability region. If $\forall S \subseteq U$, there exist certain design parameters such that $S$ is 
contained in a domain of attraction. Then, the closed loop system is said to yield a "semi-global" stability region.

PF.2 Exact output tracking - Let $\left(x_{d}(t), y_{d}(t)\right)$ for $t \in(-\infty, \infty)$ be a sufficiently smooth desired curvature in the globally fixed frame with respect to the time variable $t$. Derive a feedback control law for $F$ such that the pivot position, denoted by triplet $(t, x(t), y(t))$, of the pendulum starting from a set of initial conditions $\left(t_{0}, x\left(t_{0}\right), y\left(t_{0}\right)\right)$ converges to $\left(t, x_{d}(t), y_{d}(t)\right)$ asymptotically, i.e., $x(t)-x_{d}(t) \rightarrow 0, y(t)-y_{d}(t) \rightarrow 0$ as $t \rightarrow \infty$. Meanwhile, the pendulum is kept in $U$.

PF.3 Way-point tracking - Let $p=\left\{p_{1}, p_{2} \ldots, p_{n}\right\}$ with $p_{i}=\left(x_{r_{i}} y_{r i}\right)$ for $i=1,2, \ldots, n$ be a given sequence of points on the plane $x-y$ of the globally fixed frame. Associated with each $p_{i}$, consider the closed ball $N_{\mu i}\left(p_{i}\right)$ with center $\mathrm{p}_{i}$ and radius $\mu_{i}>0$. Derive a feedback control law for $F$ such that the pivot $(x, y)$ of the pendulum converges to $\mathrm{p}_{n}$ after visiting the ordered sequence of neighborhood $N_{\mu i}\left(p_{i}\right)$ for $i=1,2, \ldots,(n-1)$ while keeping the pendulum in the upper space $U$.

\subsection{Derivatives of the benchmark}

The system is an abstraction of many real life applications/problems (see Fig. 2)

A.1 A juggler's balancing problem - One of very childish games is to balance a pole using a finger. The pole may fall in any direction and its base moves together with the finger. When the finger moves to the left, to the right, forward or backward in a horizontal plane, a planar force $F=\left(F_{x}, F_{y}\right)$ is applied the pole to steer it around. The human's hand is replaced by a manipulator in an automated environment.

A.2 The hovering of a vector thrusted rocket - This system may hover at certain altitude either staying at a point or tracking certain trajectory. The rocket may head to any direction in a horizontal plane under the influence of injection-the main thrust. In this case, the main thrust can be decoupled to a vertical thrust against the gravity force or the drag and a planar thrust $F=\left(F_{x}, F_{y}\right)$ steering the rocket in the plane.

A.3 A personal transporter - It is a two-wheel vehicle on which a rider stands without falling over in any direction. The rider who hold the bar bending to the left, the right, forward and backward induces the cart to move intelligently to balance the rider. Some different accelerations may yielded by two wheels that together with an acceleration yielded by the centrifugal and Coriolis effects form a planar force $F=\left(F_{x}, F_{y}\right)$ to balance the rider. There is a commercial product from Segway.

A.4 The test bench - A pole with a universal joint stands on a cart sliding on a beam that in turn slides in a fixed frame. The cart and the beam that are driven by two motors respectively yields a planar force $F=\left(F_{x}, F_{y}\right)$ to the pole. This is a case where the classical inverted pendulum on the cart operates in three dimensional space;

A.5 Others - There are other controlled systems similar to the benchmark, for example, the launching of a spacecraft (without the thrust at the beginning).

As is given in A.1-A.5, a planar force $F=\left(F_{x}, F_{y}\right)$ could be derived from several different types of original actuation for different controlled systems. Without loss of generality, we take the planar force $F$ as the "generalized" force acting on the models from M.1-M.3. This gives us the same benchmark when exploring various control ideas. So, one can focus on the basic dynamic behaviors and the principles. 


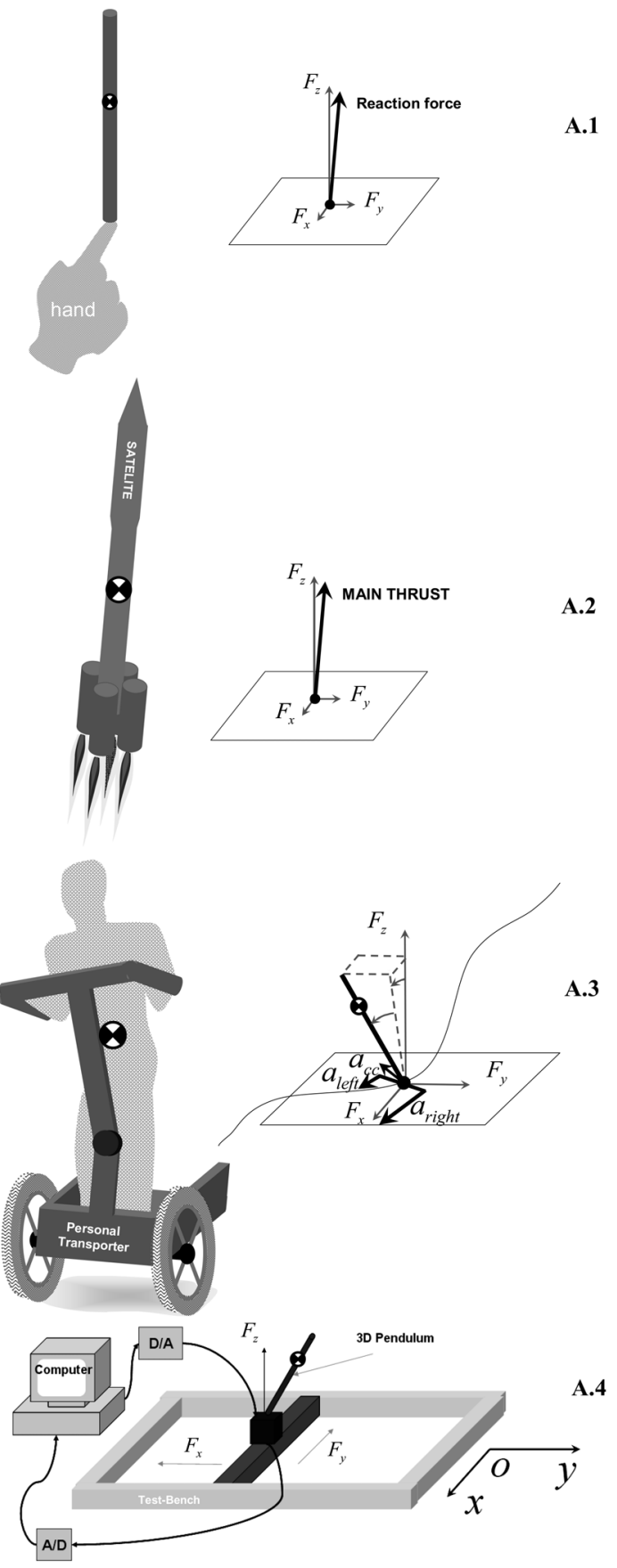

Fig. 2. Applications A.1-A.4 


\section{Nonlinear analysis and design tools}

In the realm of various nonlinear analysis and design tools, the following concepts and tools are among the mainstream (not a complete survey), which are either used, incorporated, or related to several successful designs for the benchmark

T.1 The differential geometric approach (see (Isidori, 1995)) - It is fundamental to nonlinear control systems. One of the key ideas is to transform a system to a linear one by means of feedback and coordinate transformation. The notion of "zero" dynamics plays an important role in the problem of achieving local asymptotic stability, asymptotic tracking, model matching and disturbance decoupling.

T.2 Input-to-state stability (ISS) (see (Sontag, 1990; 2005)) - The concept establishes a result on feedback redesign to obtain a desirable stability condition with respect to actuator errors, and provides a necessary and sufficiency test in terms of ISS-Lyapunov function. It brings about a number of powerful analysis tools, one of which is asymptotic "ISS" gain and its small gain theorem (Teel, 1996). The latter leads to a "celebrated" design tool-forwarding.

T.3 Forwarding and backstepping - Forwarding is a recursive control design procedure for nonlinear systems possessing an upper triangular structure. Nest saturating design (a low gain approach) (Teel, 1996) is the first tool in forwarding where design parameters are carefully selected to make the feedback interconnection of two systems satisfying small gain conditions. Lyapunov approaches (see (Mazenc \& Praly, 1996; Sepulchre et al., 1997)) for forwarding are practically very difficult to apply because constructing an "exact" cross term in the Lyapunov function is hard. Backstepping (a high gain approach) (see (Kristić, 1995; Sepulchre et al., 1997)) is a different recursive design procedure for nonlinear systems possessing a lower triangular structure. It is a very successful tool. However, one must realize that many nature systems do not possess such a structure. A misconception is that the interlaced designs (Sepulchre et al., 1997) apply also to special structures (half upper and half lower structures). Sliding mode control (see (Utkin, 1992)) can be taken as a recursive design procedure similar to backstepping.

T.4 Singular perturbations (see (Kokotović, 1986) - It is a means of taking into account neglected high-frequency phenomena and considering them in a separate fast timescale. This is achieved by treating a change in the dynamic order of a system of differential equations as a parameter perturbation, called the "singular perturbations". It results in a structure of a dynamical system with two time scales (fast and slow) so that the control problem is simplified.

T.5 Controlled Lagrangians/Hamiltanians (IDA-PBC) (see (Block et al., 2001; Ortega et al., 2002) - The methods are constructive passivity based control tools for a physical system that can be described in Lagrangian dynamics or Hamiltanian dynamics. The key notion is the energy shaping (kinetic, potential or total energy) such that the closed loop system preserves the structure of Lagrangian or Hamiltanian dynamics with a desired behavior. For example, the unstable equilibrium of the original dynamics may become a stable equilibrium of the modified dynamics. For mechanical systems, two variations are equivalent.

T.6 Stable inversion/output regulation (see (Devasia, 1996; Isidori, 1995) - The Byrnes-Isidori (see (Isidori, 1995)) regulator generalizes internal model principle to nonlinear systems that can be applied to track any trajectory generated by a given exosystem if one can 
solve the associated PDEs. The stable inversion technique (see (Devasia, 1996)) trades the requirement of solving these general PDES for a specific trajectory. Both tools can deal with the unstable "zero" dynamics that cannot be dealt with by the conventional inversion technique.

T.7 Hybrid control ${ }^{1-T h e r e ~ i s ~ n o ~ u l t i m a t e ~ d e f i n i t i o n . ~ I t ~ r e f e r s ~ t o ~ a ~ c o n t r o l ~ s y s t e m ~ t h a t ~ m i x e s ~}$ discrete parts (e.g., a controller, a supervisor) and continuous parts (e.g., a continuous plant).

\section{Constructive control designs}

\subsection{Step 1 identifying "normal" forms}

Unlike linear systems that can be written more or less in a unified manner, nonlinear systems are so diversified that one can only cope with a subclass of nonlinear systems even one particular example at a time. Therefore, nonlinear control designs are usually much more complex and difficult than linear ones. The situation well fits in with a famous sentence in Leo Tolstoy's Anna Karenina

"All happy families (linear systems here) are happy alike, all unhappy families (nonlinear systems here) are unhappy in their own way."

Nevertheless, the linear control theory is not a panacea to all control problems as it holds only around an operating point if and only if the first approximation principle holds at this point. In contrast, nonlinear control systems may yield a large (even "global") region of stability, tracks asymptotically a nonlinear trajectory that exceeds the bandwidth of a linear control system, and provides more physical insights.

A significant effort in nonlinear control designs is to identify a structure that is suitable for a particular design procedure. Ad hoc approaches for identifying a structure of a nonlinear control system maybe

- $\quad$ neglecting some nonlinear effects or considering them as perturbations;

- $\quad$ exploring physical properties to provide insight to the dynamics;

- $\quad$ taking a preliminary feedback and/or a change of states to simplify the dynamics.

Neglecting some nonlinear effects in a nonlinear design should be taken carefully because the claimed properties (e.g., a "global" domain of attraction and robustness) for the reduced dynamics may not represent a real situation. In our designs, we only neglect the disturbance and the unmodelled dynamics in analysis and design. So, we guarantee that the closed loop systems represents the original full nonlinear control system.

The structures that are explored for our designs are listed (to compare with the different structures, we abuse notations a little bit for new states)

S.1 The original dynamics maps to an "appropriate" upper triangular structure (Liu et al., 2008a)

$$
\begin{aligned}
& \dot{\zeta}_{i}=A_{i} \zeta_{i}+g_{i}\left(\xi_{i}, u\right) \quad \text { for } i=1,2,3,4 \\
& \dot{\xi}_{i}=f_{i}\left(\xi_{i}, u\right),
\end{aligned}
$$

by a nonsingular transformation $T_{1} U \rightarrow R^{8}$ (there is no constraint in new states) and a preliminary feedback $F=\alpha_{1}(\eta, u)$, where $u$ is the new input, $\xi_{i+1} \triangleq\left(\xi_{i}, \zeta_{i}\right),\left(\xi_{i}, \zeta_{i}\right)$ are the

\footnotetext{
${ }^{1}$ It does not mean a particular tool or method but a broad class of mixed tools and methods.
} 
states corresponding to each augmented subsystem and $A_{i}=0$. The feedback linearization technique (Isidori, 1995) in T.1 is incorporated.

S.2 The original dynamics also maps to two interconnected subsystems (Liu et al., 2008c)

$$
\begin{aligned}
& \dot{\omega}=A \omega+B \xi_{1}+\varphi_{\eta}\left(\xi_{1}, \xi_{2}, \zeta_{1}, \zeta_{2}\right) \\
& \dot{\xi}_{1}=\xi_{2} \\
& \dot{\xi}_{2}=u_{1} \\
& \dot{\vartheta}=A \vartheta+B \zeta_{1}+\varphi_{\vartheta}\left(\xi_{1}, \xi_{2}, \zeta_{1}, \zeta_{2}\right) \\
& \dot{\zeta}_{1}=\zeta_{2} \\
& \dot{\zeta}_{2}=u_{2}
\end{aligned}
$$

by a nonsingular transformation $T_{2} U \rightarrow R^{8}$ (there is no constraint in new states) and a preliminary feedback $F=\alpha_{2}(\eta, u)$, where $u=\left(u_{1}, u_{2}\right)$ is the new input, $\left(\xi_{1}, \xi_{2}, \omega\right)$ (with $\omega=\left(\omega_{1}, \omega_{2}\right)$ ) and $\left(\zeta_{1}, \zeta_{2}, \vartheta\right)$ (with $\vartheta=\left(\vartheta_{1}, \vartheta_{2}\right)$ ) are the states for two subsystems respectively, $A=\left(\begin{array}{ll}0 & 1 \\ 0 & 0\end{array}\right), B=\left(\begin{array}{l}0 \\ 1\end{array}\right)$, and $\varphi_{\eta}(\cdot)$ and $\varphi_{\vartheta}(\cdot)$ are interconnected terms which are high order nonlinear terms with respect to their arguments.

S.3 This structure is trivial as we can write the original unperturbed dynamics in an "appropriate" form of the Euler-Lagrangian equations (Block et al., 2001)

$$
\begin{aligned}
& \dot{\omega}=A_{u} \omega+\psi_{\eta}\left(\omega, \vartheta, u_{1}, u_{2}\right) \\
& \dot{\xi}_{1}=\xi_{2} \\
& \dot{\xi}_{2}=u_{1} \\
& \dot{\vartheta}=A_{s} \vartheta+\psi_{\vartheta}\left(\omega, \vartheta, u_{1}, u_{2}\right) \\
& \dot{\zeta}_{1}=\zeta_{2} \\
& \dot{\zeta}_{2}=u_{2}
\end{aligned}
$$

by a nonsingular transformation $T_{3} U \rightarrow \chi \in R^{8}$ ( $\chi$ is a locally bounded set about $(\omega, \vartheta) \ni 0)$ and a preliminary feedback $F=\alpha_{3}(\eta, u)$, where $u=\left(u_{1}, u_{2}\right)$ is the new input, $\left(\xi_{1}\right.$, $\left.\xi_{2}, \omega, \zeta_{1}, \zeta_{2}, \vartheta\right)$ with $\omega=\left(\omega_{1}, \omega_{2}\right)$ and $\vartheta=\left(\vartheta_{1}, \vartheta_{2}\right)$ are the new states, $A_{u}=\left(\begin{array}{ll}c & 0 \\ 0 & c\end{array}\right)$ and $A_{S}=\left(\begin{array}{cc}-c & 0 \\ 0 & -c\end{array}\right)$ for a scalar $c>0$. Here, a combination of a linear transformation and the feedback linearization technique is used.

\subsection{Step 2 applying nonlinear tools}

The structures S.1-S.4 enable us to complete a number of nonlinear control designs relatively easier for three control objectives PF.1-PF.3. Fig. 3 shows the close loop systems with the controllers NC.1-NC.5 as follows. 


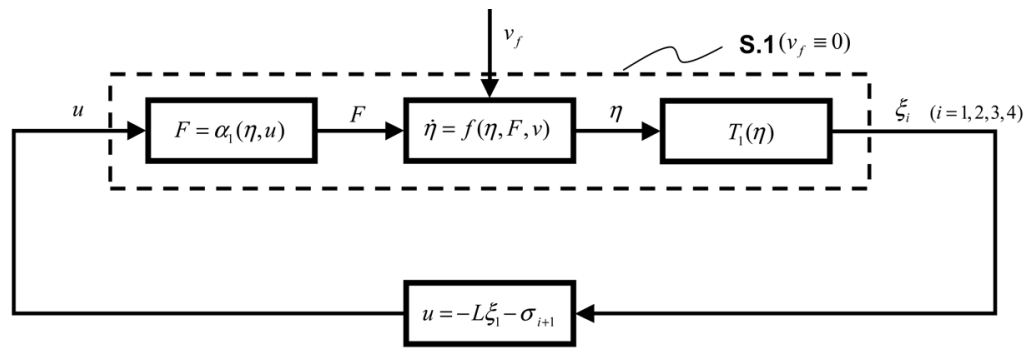

(NC.1)

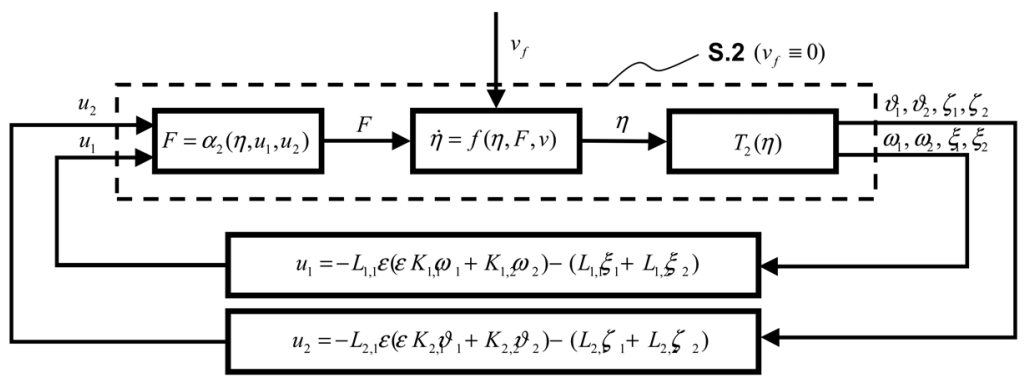

(NC.2)

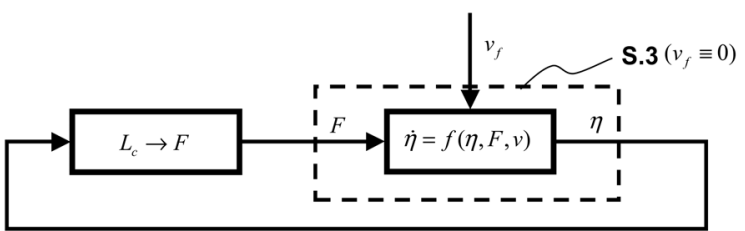

(NC.3)

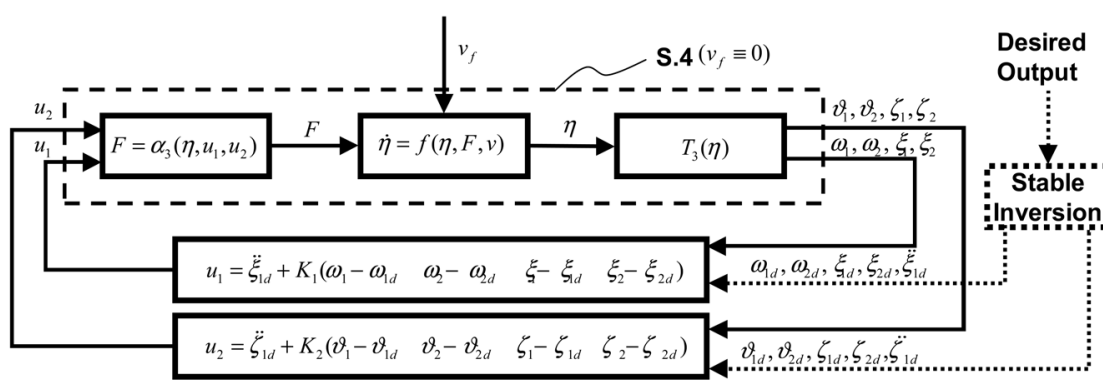

(NC.4)

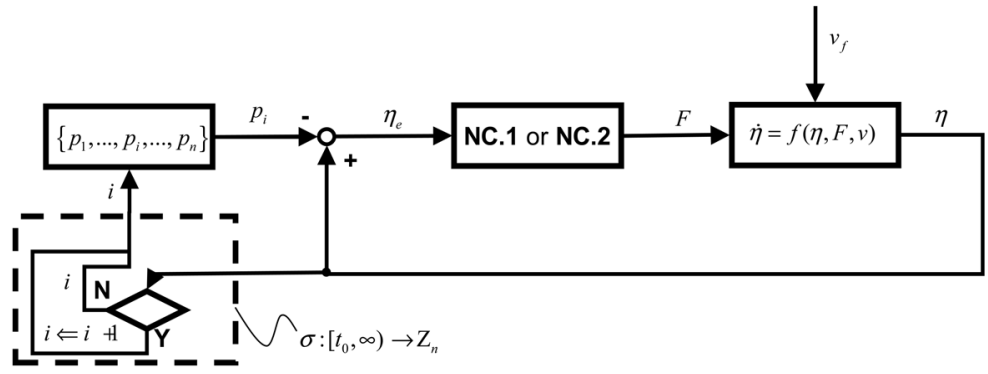

(NC.5)

Fig. 3. Diagrams of NC.1-NC.5 
NC.1 The high-low gain controller (see (Liu et al., 2008a) for PF.1 is designed on the basis of S.1

$$
u=-L \xi_{1}-\sigma_{i+1} \text { for } i=1,2,3,4
$$

where $L \in R^{4 \times 4}$ is a linear high gain matrix, $\sigma_{i+1} \triangleq \lambda_{i} \cdot \operatorname{sat}\left(\frac{1}{\lambda_{i}}\left(K_{i+1} \xi_{i+1}+\Gamma_{i+1} v_{i+1}\right)\right)$ with $v_{i+1}=\sigma_{i+2}\left(v_{5}\right.$ does not necessary to be given as the design is complete, $K_{i+1}$ and $\lambda_{i}$ are associated gain matrices and saturation levels). Nested saturating method (Teel, 1996) in T.3 is used to design a low gain control part $\sigma_{i+1}$ at the aid of a linear control design method-LQR. The controller yields a closed loop system with a "global" stability region. The design implies the existence of appropriate $\lambda_{i}$ that is related to the domain of attraction yielded by a linear controller. Practically, $\lambda_{i}$ is found by trails and errors. ISS (see (Sontag, 2005)) in T.2 is a key analysis tool in both the design and the redesign.

NC.2 The decentralized controller in (Liu et al., 2008c) for PF.1 is designed on the basis of $\mathbf{S . 2}$

$$
\begin{aligned}
u_{1}= & -\varepsilon L_{1,1}\left(\varepsilon K_{1,1} \omega_{1}+K_{1,2} \omega_{2}\right) \\
& +\left(L_{1,1} \xi_{1}+L_{1,2} \xi_{2}\right) \\
u_{2}= & -\varepsilon L_{2,1}\left(\varepsilon K_{2,1} \vartheta_{1}+K_{2,2} \vartheta_{2}\right) \\
& +\left(L_{2,1} \zeta_{1}+L_{2,2} \zeta_{2}\right)
\end{aligned}
$$

where $L_{.,}$and $K_{.,}$, are positive scalars, $\varepsilon \in(0,1)$ is time scaling parameters. The resultant closed loop system is a hidden singularly perturbed system that can be transformed into a standard singular perturbation form (slow) $\dot{\bar{x}}=f(\bar{x}, \bar{y})$, (fast) $\varepsilon \dot{y}=h(\bar{x}, \bar{y}, \varepsilon)$. A "strong" Lyapunov function comes with the design and the total stability of the system is ensured. A "semi-global" stability region (it increases as $\varepsilon$ decreases) is yielded by the closed loop system. The design is heavily relying on T.4 (see (Kokotović, 1986)).

NC.3 The controller via controlled Lagrangians in (Block et al., 2001) and (Liu et al., 2007) (a complete version) for PF.1 is based on S.3

$$
F \Leftarrow \mathcal{L}_{c}
$$

which defines a passivity based controller $F$, where $\mathcal{L}_{c}$ is defined as a controlled Lagragian that satisfies the conditions in (Block et al., 2001). Although the controller is a direct result of the theory (Block et al., 2001) in T.5, the derivation is technically complex. A "weak" Lyapunov function comes with the design, that is, an energy function of the closed loop system. LaShall's invariance principle is used to established the stability but the principle cannot guarantee the stability under disturbances.

NC.4 The exact output tracking controller in (Liu et al., 2008b) for PF.2 is a designed on the basis of $\mathbf{S . 3}$

$$
\begin{array}{cc}
u_{1}=\ddot{\xi}_{1 d}+K_{1} & \left(\omega_{1}-\omega_{1 d} \omega_{2}-\omega_{2 d}\right. \\
& \left.\xi_{1}-\xi_{1 d} \xi_{2}-\xi_{2 d}\right) \\
u_{2}=\ddot{\zeta}_{1 d}+K_{2} & \left(\vartheta_{1}-\vartheta_{1 d} \vartheta_{2}-\vartheta_{2 d}\right. \\
& \left.\zeta_{1}-\zeta_{1 d} \zeta_{2}-\zeta_{2 d}\right)
\end{array}
$$


where $\left\{\xi_{1 d}, \zeta_{1 d} \mid \xi_{2 d}, \zeta_{2 d}, \ddot{\xi}_{1 d}, \ddot{\zeta}_{1 d}, \omega_{1 d}, \omega_{2 d}, \vartheta_{1 d}, \vartheta_{2 d}\right\}$ are obtained based on the stable inversion tool (Devasia, 1996) in T.6 with respect to a desired output trajectory. K. are linear feedback gain matrices obtained by a linear controller design-LQR. $\left(\ddot{\xi}_{1 d}, \ddot{\zeta}_{1 d}\right)$ is a guidance controller (a feedforward part) and the rest is a feedback minimizing the tracking errors and rejecting exogenous disturbances. For an achievable desired trajectory that is $c_{2}(-\infty, \infty)$, the output (the translational variables $\xi_{1}$ and $\zeta_{1}$ - the original $x$ and $y$ ) of the closed loop system tracks exactly the desired trajectory while keeping the pendulum upward.

NC.5 The hybrid controller in (Liu \& Yang, 2010) for PF.3 is in the category of T.7. The result is relying on NC.1 or NC.2 and an event driven piecewise constant signal $\sigma\left[t_{0}, \infty\right) \rightarrow \mathbf{Z}_{n}$ that is continuous from the right at every point and is defined recursively by

$$
\sigma=\bar{\alpha}\left(\chi, \psi, \sigma^{-}\right), \quad t \geq t_{0}
$$

where $\chi$ and $\psi$ are metrics on the current tracking errors with respect to the neighborhood $N_{\mu i}$ of $i$ th way-point, $\sigma(\tau)$ is equal to the limit from the left of $\sigma(\tau)$ as $\tau \rightarrow$ $t$ based on an event that determines the discrete value $i$ in a set $\{1,2, \ldots, n\}$. The controller yields either "global" or "semi-global" stability region to the closed loop system inherit from NC.1 or NC.2. The ordered sequence of way-points are guaranteed but the timing to a way-point is uncertain.

\section{Conclusion and future work}

The cutting-edge theoretical nonlinear analysis and designs tools have been used successfully to solve the challenging control goals for a four degrees of freedom spherical inverted pendulum, such as the global stabilization and the nonlinear exact tracking. However, the tools are unable to yield satisfactory controllers on their own. A designer should perform preliminary designs via identify the special structures, "normal" forms, to bridge the gap. Observed from these successful designs, a good insight to the physical dynamical system would help us to find a way, bridging the gap. The experiences obtained from the benchmark example should be extended to other nonlinear control systems. Techniques of identifying various "normal forms" should be emphasized.

\section{References}

Albouy, X. \& Praly, L. (2000). On the use of dynamic invariants and forwarding for swinging up a spherical inverted pendulum, in Proceedings of 39th Conference on Decision \& Control, Sydney, Australia, pp. 1667-1672.

Bloch, A.; Chang, D.; Leonard, N. \& Marsden, J. (2001). Controlled Lagragians and the stabilization of mechanical systems II potential shaping, IEEE Transactions on Automatic Control, Vol. 41, pp. 1556-1571.

Devasia, D.; Chen, D. \& Paden, B. (1996). Nonliear inversion-based output tracking, IEEE Transactions on Automatic Control, Vol. 41, pp. 930-942.

Isidori, A. (1995). Nonlinear Control System (3rd edition), Springer.

Kokotović; P. Khalil, H. \& O'Reilly, J. (1986). Singular Perturbation Methods in Control Analysis and Design, Academic Press Inc.. 
Krstić M.; Kanellakopoulos, L. \& Kokotović, P. (1995). Nonlinear and Adaptive Control Design, John Wiley \& Sons.

Liu, G. (2006). Modeling, Stabilizing Control and Trajectory Tracking of a Spherical Inverted Pendulu. Ph.D Thesis, The University of Melbourne.

Liu, G.; Challa, I. \& Yu, L.(2007). Revisit controlled Lagrangians for spherical inverted pendulum , International Journal of Mathematics and Computers in Simulation, Vol. 1, No. 1, pp. 209-214.

Liu, G.; Mareels, I. \& Nešić, D. (2008). Decentralized control design of interconnected chains of integrators a case study, Automatica, Vol. 44, No. 8, pp. 2171-2178.

Liu, G.; D. Nešić \& I. Mareels (2008). Nonlinear stable-inversion based output tracking for the spherical inverted pendulum, International Journal of Control, Vol. 81, No.7, pp. 1035-1053.

Liu, G.; D. Nešić \& I. Mareels (2008). Nonlinear stable-inversion based output tracking for the spherical inverted pendulum, International Journal of Control, Vol. 81, No.1, pp. 116-133.

Liu, G. \& Yang, R. (2010). Minimizing operating points of way point tracking of an unstable nonlinear plant, Asian Journal of Control, Vol. 12, No. 1, pp. 84-88.

Mazenc, F. \& Praly, L. (1996). Adding integrations, saturated controls, and stabilization for feedforward systems, IEEE Transactions on Automatic Control, Vol.41, pp. 1559-1577.

Ortega, R.; Spong, W.; Gomez-Estern, F. \& Blankenstein, G. (2002). Stabilization of a class of underactuated mechanical systems via interconnection and damping assignment. IEEE Transactions on Automatic Control, Vol. 47, pp. 1218-1233.

Sepulchre, R.; Janković, M. \& Kokotović, P. (1997). Constructive Nonlinear Control, Springer, pp. 979-984.

Sepulchre, R.; Janković M. \& Kokotović, P. (1997). Integrator forwarding a new recursive nonlinear robust design. Automatica, Vol. 393, pp. 979-984.

Sontag, E. (1990). Further facts about input to state stabilization. IEEE Transactions on Automatic Control, Vol. 35, pp. 473-476.

Sontag, E. (2005). Input to state stability Basic concepts and results, Springer Lecture Notes in Mathematics, Springer.

Teel, A. (1996). A nonlinear small gain theorem for the analysis of control systems with saturation. IEEE Transactions on Automatic Control, Vol. 41, pp. 1256-1270.

Utkin, V. (1992). Sliding modes in control optimization, Springer-Verlag. 


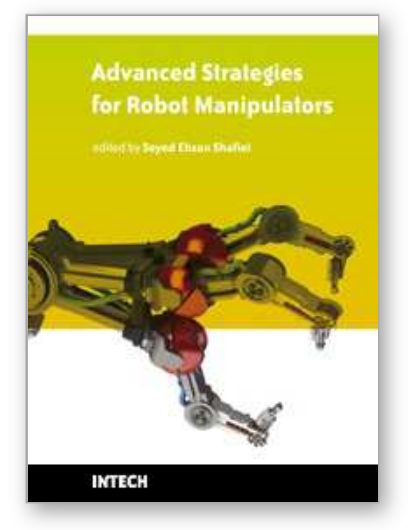

\author{
Advanced Strategies for Robot Manipulators \\ Edited by S. Ehsan Shafiei
}

ISBN 978-953-307-099-5

Hard cover, 428 pages

Publisher Sciyo

Published online 12, August, 2010

Published in print edition August, 2010

Amongst the robotic systems, robot manipulators have proven themselves to be of increasing importance and are widely adopted to substitute for human in repetitive and/or hazardous tasks. Modern manipulators are designed complicatedly and need to do more precise, crucial and critical tasks. So, the simple traditional control methods cannot be efficient, and advanced control strategies with considering special constraints are needed to establish. In spite of the fact that groundbreaking researches have been carried out in this realm until now, there are still many novel aspects which have to be explored.

\title{
How to reference
}

In order to correctly reference this scholarly work, feel free to copy and paste the following:

Guangyu Liu and Yanxin Zhang (2010). On Nonlinear Control Perspectives of a Challenging Benchmark, Advanced Strategies for Robot Manipulators, S. Ehsan Shafiei (Ed.), ISBN: 978-953-307-099-5, InTech, Available from: http://www.intechopen.com/books/advanced-strategies-for-robot-manipulators/on-nonlinearcontrol-perspectives-of-a-challenging-benchmark

\section{INTECH}

open science | open minds

\section{InTech Europe}

University Campus STeP Ri

Slavka Krautzeka 83/A

51000 Rijeka, Croatia

Phone: +385 (51) 770447

Fax: +385 (51) 686166

www.intechopen.com

\section{InTech China}

Unit 405, Office Block, Hotel Equatorial Shanghai

No.65, Yan An Road (West), Shanghai, 200040, China

中国上海市延安西路65号上海国际贵都大饭店办公楼 405 单元

Phone: +86-21-62489820

Fax: +86-21-62489821 
(C) 2010 The Author(s). Licensee IntechOpen. This chapter is distributed under the terms of the Creative Commons Attribution-NonCommercialShareAlike-3.0 License, which permits use, distribution and reproduction for non-commercial purposes, provided the original is properly cited and derivative works building on this content are distributed under the same license. 